\section{Increase in detection of early RCC might improve survival}

In the US, evidence indicates that newly diagnosed renal cell carcinoma (RCC) is more likely to be low-stage disease today than formerly. The effect of this trend towards a lower stage of RCC at diagnosis, however, requires evaluation. Kane and colleagues, therefore, used data from the National Cancer Data Base to reveal changes in RCC stage distribution over time, and to determine whether these changes influenced patients' outcomes.

Data were analyzed from 205,963 patients diagnosed with RCC between 1993 and 2004 for whom information on RCC stage at diagnosis was available. Of their tumors, $50.6 \%$ were stage I, $26.7 \%$ were stage II or III, and $22.7 \%$ were stage IV. Over a 12-year period, the percentage of patients with stage I tumors at diagnosis increased from $43.0 \%$ to $57.1 \%$, counterbalanced by a decreased percentage of patients diagnosed with stage II-IV tumors (e.g. the proportion of stage IV tumors decreased from $27.4 \%$ to $18.7 \%$ ). The average size of stage I tumors at detection decreased from $4.1 \mathrm{~cm}$ to $3.6 \mathrm{~cm}$ over this period. The observed downward stage migration resulted in a small but significant improvement in overall 5-year survival $(63.3 \%$ of patients diagnosed in 1993 survived for 5 years, compared with $66.6 \%$ of patients diagnosed in 1998).

This study confirmed that stage migration-a shift in the distribution of RCC stages at presentation-has occurred in the US, primarily because of incidental detection of RCCs during abdominal imaging studies for non-RCC indications.

Original article Kane CJ et al. (2008) Renal cell cancer stage migration: analysis of the National Cancer Data Base. Cancer 113: $78-83$

\section{Vitamin D concentration does not affect the risk of prostate cancer}

Some studies have indicated that high levels of circulating vitamin D might reduce prostate cancer risk or improve outcomes of patients with this malignancy; epidemiological studies, however, have yet to provide conclusive results. Prostate cells convert the circulating form of the vitamin -25-hydroxyvitamin D-to biologically active 1,25-dihydroxyvitamin $D$, which, at high concentrations, inhibits proliferation and differentiation of prostate cancer cell lines. Ahn and colleagues studied whether increased serum levels of 25-hydroxyvitamin D are associated with improved outcomes in men screened for prostate cancer.

The researchers assessed data from 749 men with prostate cancer and 781 men without prostate cancer who participated in the US-based Prostate, Lung, Colorectal, and Ovarian Cancer Screening Trial. Serum vitamin $\mathrm{D}$ concentrations were measured in blood samples taken at enrollment; all prostate cancer cases included in this analysis were diagnosed 1-8 years after the blood draw.

The researchers found no statistically significant difference in the risk of prostate cancer with increasing vitamin $\mathrm{D}$ concentration. They did see some evidence of an increased risk of aggressive disease (stage III or IV tumors or a Gleason sum $\geq 7$ ) associated with high vitamin $D$ concentrations, but this trend was not statistically significant and the association did not show a linear dose-response relationship.

These results do not support the hypothesis that increased serum vitamin $D$ levels decrease the risk of prostate cancer. Further studies are required to confirm these results and clarify the effects of vitamin $D$ on prostate cancer.

Original article Ahn J et al. (2008) Serum vitamin D concentration and prostate cancer risk: a nested case-control study. J Natl Cancer Inst 100: 796-804

\section{Erectile dysfunction predicts coronary heart disease in patients with type 2 diabetes}

Erectile dysfunction (ED) occurs more often in men with diabetes than in the general population, and is also associated with atherosclerosis. Ma et al. have found that ED is associated with subsequent occurrence of coronary heart disease (CHD) events in Chinese men with type 2 diabetes who initially have no clinical manifestations of CHD, and suggest that ED could be used as a surrogate marker to predict future CHD.

This prospective study enrolled 2,306 consecutive men with type 2 diabetes and without clinical evidence of stroke, CHD or peripheral artery disease. All patients underwent a 